\title{
Consumo de álcool, tabaco e outras drogas entre adolescentes do ensino fundamental de um município brasileiro
}

\author{
Consumption of alcohol, tobacco and other drugs among adolescents from an elementary \\ school in a brazilian city \\ Consumo de alcohol, tabaco y otras drogas entre adolescentes de la escuela primaria en \\ un municipio brasileño
}

Rodrigo Eurípedes da Silveira*; Álvaro da Silva Santos**; Gilberto de Araújo Pereira***

\section{Resumo}

Enquadramento: O consumo de drogas entre adolescentes é premente preocupação para a saúde pública em nível mundial. Objetivos: Avaliar o consumo de álcool, tabaco e drogas ilícitas entre estudantes das $7^{\mathrm{a}} \mathrm{s}$ e $8^{\mathrm{a}} \mathrm{s}$ séries de uma escola municipal de Uberaba, Brasil.

Metodologia: Estudo exploratório de corte transversal. Amostra de 189 estudantes. Questionário semiestruturado, auto aplicável. Resultados: Predominou o sexo masculino (52,9\%), com idade média de 14,3 $\pm 1,1$ anos e rendimento familiar entre 1 a 3 salários mínimos (88,9\%). O consumo de álcool foi significativamente superior nos aprovados na escola $(84,2 \% ; p<0,0001)$ e naqueles com rendimento superior a três sálarios minimos $(100 \% ; p<0,0001)$. O consumo de tabaco foi associado à $7^{\mathrm{a}}$ série $(79,5 \% ; p=0,0004)$ e o rendimento inferior a um salário mínimo ( $67,5 \%$; $<<0,0001)$. Quanto ao consumo de drogas ilícitas houve predominio significativo somente naqueles com rendimento superior a três salários minímos $(66,7 \% ; \mathrm{p}=0,0176)$.

Conclusão: Esta pesquisa mostra a necessidade de ações de educação em saúde para adolescentes na temática álcool e drogas, bem como uma melhor e mais efetiva educação permanente de seus professores.

Palavras-chave: adolescente; bebidas alcoólicas; hábito de fumar; drogas ilícitas.

\begin{abstract}
Background: Drug use among adolescents is an urgent public health concern worldwide.

Objectives: To assess the consumption of alcohol, tobacco and illicit drugs among 7 th and 8th graders of a municipal school in Uberaba, Brazil.

Methodology: Cross-sectional exploratory study. Sample of 189 students. Semi-structured and self-administered questionnaire.

Results: Most students were boys (52.9\%), with a mean age of $14.3 \pm 1.1$ years and a household income between 1 and 3 minimum wages (88.9\%). Alcohol consumption was significantly higher among approved students $(84.2 \% ; \mathrm{p}<0.0001)$ and among those with incomes above three minimum wages $(100 \% ; p<0.0001)$. Tobacco consumption was associated with the 7 th grade $(79.5 \%$; $\mathrm{p}=0.0004)$ and incomes inferior to one minimum wage $(67.5 \% ; \mathrm{p}<$ 0.0001). There was a significant predominance of illicit drug use only among students with incomes above three minimum wages $(66.7 \%$; $\mathrm{p}=0.0176$ ).

Conclusion: This study points out the need for health education interventions related to alcohol and drugs among adolescents, as well as a better and more effective continuous training of their teachers.
\end{abstract}

Keywords: adolescent; alcoholic beverages; smoking habit; illicit drugs.

\footnotetext{
* Enfermeiro. Mestrando em Ciências da Saúde pelo Instituto de Assistência Médica ao Servidor Público Fstadual de São Paulo (IAMSPE). Professor Auxiliar da Universidade Federal do Triângulo Mineiro (UFTM). Morada para correspondência: Rua Campos Sales, 840. B. Abadia. Uberaba-MG. CEP: 38026-260 [rodrigo_euripedes@hotmail.com].

38026-260 [rodrigo_euripedes@ hotmail.com]. Departamento de Educação e Enfermagem em Saúde Comunitária e Mestrado em Atenção em Saúde da UFTM [alvaroenf@hotmail.com].

*** Bacharel em Estatística. Doutor em Estatística pela Universidade Federal de São Carlos. Professor adjunto do Departamento de Educação e Enfermagem em Saúde Comunitária da UFTM [pereira gilberto@yahoo.com.br].
}

\section{Resumen}

Marco contextual: El consumo de drogas entre adolescentes es un problema de salud pública relevante en todo el mundo. Objetivos: La presente investigación tiene como objetivo evaluar el consumo de alcohol, tabaco y drogas ilícitas entre estudiantes de los cursos séptimo y octavo de una escuela municipal de Uberaba, Brasil.

Metodología: Estudio exploratorio y transversal. Muestra de 189 estudiantes. Cuestionario semiestructurado autoaplicable. Resultados: Predominaron los varones (52,9\%), con una edad media de $14,3 \pm 1,1$ años y una renta familiar entre 1 y 3 salarios mínimos (88,9\%). El consumo de alcohol fue significativamente superior en aquellos aprobados en la escuela $(84,2 \% ; p<0,0001)$ y con una renta superior tres salarios mínimos $(100 \% ; p<0,0001)$. El consumo de tabaco fue más frecuente en el séptimo curso $(79,5 \% ; p=0,0004)$ y en aquellos con una renta inferior a un salario mínimo $(67,5 \% ; p<0,0001)$. En cuanto al consumo de drogas ilícitas, se asoció a los jóvenes con una renta superior a tres salarios mínimos (66,7\%; $p=0,0176)$.

Conclusión: Esta investigación muestra la necesidad de realizar acciones de educación para la salud para adolescentes enfocadas en los temas alcohol y drogas, así como una educación permanente de sus profesores mejor y más eficaz.

Palabras clave: adolescente; bebidas alcohólicas; hábito de fumar; drogas ilícitas.

Recebido para publicação em: 28.08 .12

Aceite para publicação em: 06.05.14 


\section{Introduçáo}

Este estudo apresenta os resultados de uma pesquisa vinculada ao Projeto de Extensão "Protagonismo Juvenil como estratégia de Intervenção Familiar e Comunitária no Residencial 2000", que teve apoio da Fundação de Ensino e Pesquisa de Uberaba, Minas Gerais, Brasil (FUNEPU), na perspectiva de aproximar as fronteiras da Universidade para a Educação e Investigação da Saúde em âmbito escolar.

No decorrer das ações de Extensão, observaram-se inúmeras situações de risco aos quais os adolescentes daquela comunidade estavam envolvidos, com o consumo de drogas lícitas e ilícitas dentro e fora da escola. Para além disso, situações adversas de relacionamento interpessoal e condições de vida dentro e fora da sala de aula instigou os pesquisadores à seguinte indagação: "Qual o perfil de utilização de drogas lícitas e ilícitas entre os adolescentes do ensino fundamental?"

A adolescência, entendida como uma das fases mais importantes do desenvolvimento humano, guarda consigo inúmeras situações em que o indivíduo se abre a novas experiências em busca da sua identidade e expressão, além de situações que thes dêem a sensação de liberdade. É a fase de descoberta e afirmação da sua identidade de género e outras funções como erotismo, prazer, intimidade e reprodução, concomitantes a alterações biológicas, psicológicas e sociais (Silveira, Reis, Santos, \& Borges, 2011).

Por outro lado, as indústrias de substâncias psicoativas investem muito em espaços de difusão da oferta e da sedução ao consumo dos seus produtos - as drogas lícitas - como as bebidas alcóolicas e o cigarro, atraindo a atenção de milhares de jovens em busca de prazer. Ainda, há que se considerar a inserção em grande escala das drogas ilícitas no Brasil, estando mais disponível nas comunidades carentes, de fácil acesso a grupos em situações de risco e vulnerabilidade, como a população adolescente (Jesus, Lima, Martins, Matos, \& Souza, 2011).

Neste sentido, é fundamental a identificação e a quantificação destas situações de risco, procurando explicitar ainda, os fatores determinantes ou associados a estes comportamentos, para orientar ações interventivas nos campos da prevenção e do tratamento dos problemas decorrentes do uso de substâncias psicoativas, hoje ainda deficientes.

\section{Objetivo}

A presente investigação pretende identificar e analisar o consumo de álcool, tabaco e drogas ilícitas entre adolescentes, relacionados com o sexo, série, reprovação escolar e rendimento familiar de estudantes de $7^{\mathrm{a}} \mathrm{S}$ e $8^{\mathrm{a}} \mathrm{s}$ séries de uma escola municipal de Uberaba, Minas Gerais, Brasil.

\section{Enquadramento}

A alta prevalência e as consequências associadas ao consumo de drogas lícitas e ilícitas entre adolescentes têm estimulado a realização de diversos estudos em todos os continentes povoados do planeta, apresentando elevada inserção do álcool, do tabaco e de outras drogas entre a população jovem, como em estudos realizados na Espanha (Inglés et al., 2007), no Japão (Suzuki, Kimura, Takeda, \& Matsushita, 2008), na África do Sul (Naude, Senekal, Laubscher, Carey, \& Fein, 2011) e na Austrália e Estados Unidos (Coomber, Toumbourou, Miller, Staiger, Hemphill, \& Catalano, 2012).

Nas últimas décadas, o fenómeno do uso de drogas alcançou extraordinária importância pela sua difusão, pelo consumo em larga escala pelos jovens e por suas consequências sociais e sanitárias, além do incremento da criminalidade e violência. No contexto do imaginário adolescente, as drogas são mecanismos que favorecem a socialização e o bem-estar, além de propiciar uma sensação de liberdade e alívio das suas preocupações e medos. Em contrapartida, as consequências cobram um preço alto, acarretando uma série de problemas físicos, psicológicos e sociais, podendo acabar por consumir até mesmo a vida deste adolescente (Jinez, Souza, \& Pillón, 2009).

Assim como o uso de drogas ilícitas, o álcool também constitui uma das principais causas desencadeadoras de situações de vulnerabilidade na adolescência, e que segundo a Organização Mundial da Saúde (OMS) trata da substância psicoativa mais consumida no mundo e representa a principal droga de escolha entre crianças e adolescentes. No Brasil, o álcool é a droga mais utilizada pela população adulta e o seu consumo tem aumentado entre os jovens nas últimas décadas, sobretudo entre aqueles com 12 a 15 anos de idade (Horta, Horta, Pinheiro, Morales, \& Strey, 2007). 
O consumo de bebidas alcoólicas pode estar associado ao consumo de tabaco e ao comportamento sexual de risco, condicionando situações de vulnerabilidade para os adolescentes, que dificultam a atuação das entidades de proteção social, quando o jovem não possui um suporte familiar adequado (Pedrosa, Camacho, Passos, \& Olveira, 2011; Silveira \& Santos, 2012). Estudos recentes apontam que não há diferenças entre o consumo destas substâncias entre géneros, ou seja, há uma tendência de paridade entre o consumo de drogas entre meninos e meninas, como no estudo que considerou 971 adolescentes entre 10 e 18 anos, dos quais 55\% eram do sexo masculino, 33,8\% relataram ter feito uso de bebidas alcoólicas no último mês, 13,5\% de cigarro e 6,4\% de drogas ilícitas (Malbergier, Cardoso, Amaral, \& Santos, 2012).

Afirma-se que residir com os pais, tendo apoio e supervisão dos mesmos e compartilhando de momentos de lazer, refeições e convívio tem efeito protetor nos hábitos de fumar, beber e usar drogas. Em pesquisa de proporção nacional, com mais de 60 mil estudantes brasileiros, observou-se que os alunos que faltam às aulas sem avisar os pais têm maior probablidade de fumar, beber e experimentar drogas, cujo consumo tem sido associado à avaliação negativa da relação familiar, à falta de suporte/monitoramento e ao uso de substâncias pelos familiares destes adolescentes (Malta et al., 2011; Malbergier et al., 2012).

A presente investigação foi estruturada a considerar as perspetivas já apresentadas, somadas às características da realidade local, em que ocorrem inúmeras situações de violência e vulnerabilidade social, estrutural e psicológica no âmbito familiar. Destaca-se ainda carências estruturais no âmbito educacional da referida comunidade, como a falta de um local para convívio, de espaço para desportos e lazer, e por um quadro de professores constantemente expostos a fatores stressantes, associados aos contextos sociais e económicos (Silveira, et al., 2011).

\section{Metodologia}

Estudo prospetivo e exploratório de corte transversal, com amostragem de conveniência, que incluiu todos os alunos das $7^{\mathrm{a}} \mathrm{s}$ e $8^{\mathrm{a}} \mathrm{s}$ séries de uma Escola Pública Municipal, em Uberaba-MG. Dos 203 estudantes, 189 foram incluídos na pesquisa, tendo devolvido o
Termo de Consentimento Livre e Esclarecido assinado pelos seus responsáveis, uma vez que eram menores de 18 anos.

A colheita de dados ocorreu entre setembro de 2010 e março de 2011, recorrendo a um questionário semiestruturado e autoaplicável sobre comportamentos em saúde, previamente ajustado por um teste piloto numa escola diferente daquela que foi objeto da pesquisa.

As variáveis de interesse foram: uso, frequência e idade de início quanto à bebida alcoólica, tabaco e drogas ilícitas. Considerou-se como possíveis variáveis explicativas a idade, sexo, série, reprovação escolar e rendimento familiar.

Os dados foram analisados pelo programa Statistica 6.0. Primeiramente utilizou-se a análise descritiva das quais as variáveis categóricas foram apuradas quanto às frequências absolutas, percentuais e numéricas, a partir das medidas de centralidade e dispersão. Para analisar as associações de interesse entre variáveis categóricas foram utilizados o teste qui-quadrado e cálculo do Odds-Ratio (OR). Quanto à comparação da idade de inicio do uso de drogas lícitas e ilícitas foi utilizado ANOVA-F. Para todos os testes foi considerado nível de significância de $5 \%(p<0,05)$ (Agresti, 2007; Govindarajuju, 2007).

A presente investigação foi submetida ao Comitê de Ética em Pesquisa com Seres Humanos da Universidade Federal do Triângulo Mineiro e aprovada pelo protocolo $\mathrm{n}^{0} 1764 / 2011$, e contou ainda com anuência da direção da escola em que se realizou o estudo.

\section{Resultados}

A amostra do estudo foi composta por 189 adolescentes sendo 100 (52,9\%) do sexo masculino e $89(47,1 \%)$ do sexo feminino. Dentre os adolescentes do sexo masculino 52 (52,0\%) estavam na $7^{\mathrm{a}}$ série, 31 (31\%) já haviam sido reprovados, 40 (40,0\%) com rendimento familiar abaixo de um salário mínimo e padrão médio de idade de 14,3 $\pm 1,2$ anos, enquanto no sexo feminino 55 (61,8\%) estavam na $8^{a}$ série, 35 (39,3\%) já haviam sido reprovados, 33 (37,1\%) com rendimento familiar abaixo de um salário mínimo e padrão médio de idade de 14,3 $\pm 1,0$ anos, como se observa na Tabela 1. 
Tabela 1

Distribuição dos adolescentes de uma escola pública quanto a série, reprovação escolar, rendimento familiar e idade, Uberaba, 2011.

\begin{tabular}{|c|c|c|c|c|c|}
\hline & & \multicolumn{2}{|c|}{ Masculino } & \multicolumn{2}{|c|}{ Feminino } \\
\hline & & $\mathrm{n}$ & $\%$ & $\mathrm{~N}$ & $\%$ \\
\hline \multirow[t]{2}{*}{ Série } & $7^{\mathrm{a}}$ série & 52 & 52,0 & 34 & 38,2 \\
\hline & $8^{a}$ série & 48 & 48,0 & 55 & 61,8 \\
\hline \multirow[t]{2}{*}{ Reprovação Escolar } & Sim & 31 & 31,0 & 35 & 39,3 \\
\hline & Não & 69 & 69,0 & 54 & 60,7 \\
\hline \multirow[t]{3}{*}{ Rendimento Familiar (em SM*) } & $\leq 1 \mathrm{SM}$ & 40 & 40,0 & 33 & 37,1 \\
\hline & 1 a 2 SM & 43 & 43,0 & 52 & 58,4 \\
\hline & $\geq 3 \mathrm{SM}$ & 17 & 17,0 & 4 & 4,5 \\
\hline \multirow[t]{5}{*}{ Idade (anos) } & 13 & 27 & 27,0 & 23 & 25,8 \\
\hline & 14 & 43 & 43,0 & 30 & 33,7 \\
\hline & 15 & 15 & 15,0 & 27 & 30,3 \\
\hline & 16 & 6 & 6,0 & 8 & 9,0 \\
\hline & 17 & 9 & 9,0 & 1 & 1,1 \\
\hline Total & & 100 & 100 & 89 & 100 \\
\hline
\end{tabular}

SM: Salário Mínimo, na época da pesquisa equivalente a $\mathrm{R} \$ 540,00$, aproximadamente $€ 180,00$.

O consumo de álcool, cigarro e drogas ilícitas em relação ao sexo, série, reprovação escolar e rendimento familiar é apresentado na Tabela 2. Dos 115 respondentes sobre a utilização de bebidas alcoólicas, afirmaram ter consumido bebidas alcoólicas: a) 66 (77,7\%) dos 85 adolescentes do sexo masculino e 49 (63,6\%) das 77 adolescentes do sexo feminino; b) 76 (80,9\%) dos 94 adolescentes na $8^{\mathrm{a}}$ série e $39(57,4 \%)$ dos 68 na $7^{\mathrm{a}}$ série, sendo esta associação significativa $(p=0,0011)$ apresentando uma probablidade de consumo de álcool na $8^{a}$ série de 3,1 vezes a probablidade na $7^{\mathrm{a}}$ série $(\mathrm{OR}=3,1)$; (c) 96 (84,2\%) dos 114 adolescentes sem reprovação escolar e 19 (39,6\%) com reprovação, demonstrando uma associação significativa $(\mathrm{p}<0,0001)$ sendo a probablidade de consumo de álcool nos adolescentes aprovados 8,1 vezes a probablidade nos reprovados $(\mathrm{OR}=8,1)$ e (d) 21 (100\%) dos adolescentes com rendimento familiar igual ou superior a três salários minimos, 69 (78,4\%) com um rendimento entre 1 e 2 salários mínimos e 25 (47,2\%) com rendimento inferior a 1 salário, demonstrando neste caso uma associação significativa $(p<0,0001)$ sendo a probablidade de consumo de bebidas alcoólicas maior naqueles com maior rendimento familiar, como se observa na Tabela 2.

Tabela 2

Distribuição dos adolescentes de uma escola pública referente ao uso de bebida alcoolica, tabaco e drogras ilícitas em relação ao sexo, série, reprovação escolar e rendimento familiar. Uberaba, 2011.

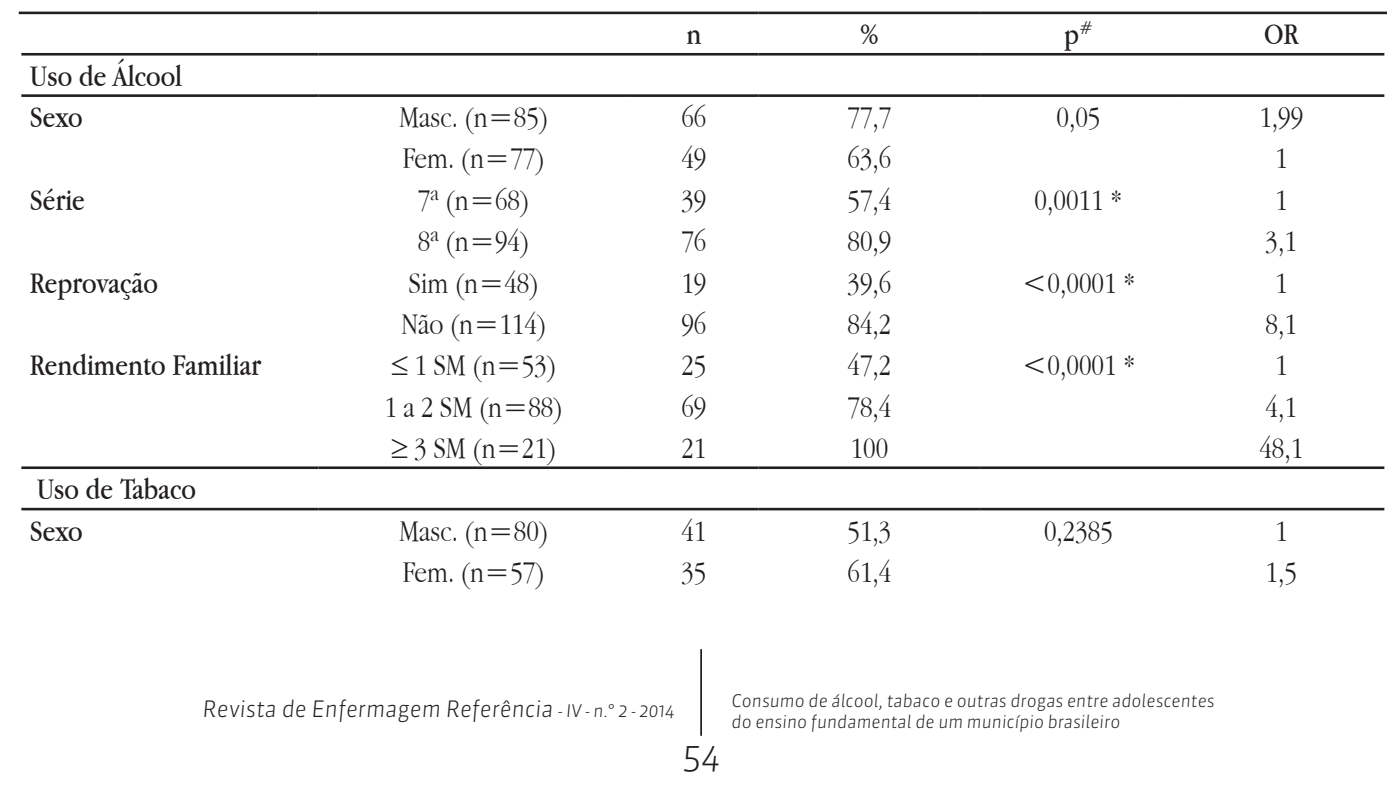




\begin{tabular}{|c|c|c|c|c|c|}
\hline \multirow[t]{2}{*}{ Série } & $7^{a}(n=39)$ & 31 & 79,5 & $0,0004 *$ & 4,6 \\
\hline & $8^{\mathrm{a}}(\mathrm{n}=98)$ & 45 & 45,9 & & 1 \\
\hline \multirow[t]{2}{*}{ Reprovação } & $\operatorname{Sim}(n=19)$ & 10 & 52,6 & 0,7882 & 1 \\
\hline & Não (n=118) & 66 & 55,9 & & 1,1 \\
\hline \multirow[t]{3}{*}{ Rendimento Familiar } & $\leq 1 \mathrm{SM}(\mathrm{n}=40)$ & 27 & 67,5 & $<0,0001 *$ & 71,3 \\
\hline & $1 \mathrm{a} 2 \mathrm{SM}(\mathrm{n}=80)$ & 49 & 61,3 & & 55 \\
\hline & $\geq 3 \mathrm{SM}(\mathrm{n}=17)$ & 0 & 0 & & 1 \\
\hline \multicolumn{6}{|l|}{ Uso de Drogas Ilícitas } \\
\hline \multirow[t]{2}{*}{ Sexo } & Masc. $(n=81)$ & 38 & 46,9 & 0,0542 & 1,9 \\
\hline & Fem. $(n=67)$ & 21 & 31,3 & & 1 \\
\hline \multirow[t]{2}{*}{ Série } & $7^{\mathrm{a}}(\mathrm{n}=59)$ & 22 & 37,3 & 0,6021 & 1 \\
\hline & $8^{a}(\mathrm{n}=89)$ & 37 & 41,6 & & 1,2 \\
\hline \multirow[t]{2}{*}{ Reprovação } & $\operatorname{Sim}(n=46)$ & 14 & 30,4 & 0,1156 & 1 \\
\hline & Não $(n=102)$ & 45 & 44,1 & & 1,8 \\
\hline \multirow[t]{3}{*}{ Rendimento Familiar } & $\leq 1 \mathrm{SM}(\mathrm{n}=46)$ & 14 & 30,4 & $0,0176 *$ & 1 \\
\hline & 1 a $2 S M(n=81)$ & 31 & 38,3 & & 1,4 \\
\hline & $\geq 3 \mathrm{SM}(\mathrm{n}=21)$ & 14 & 66,7 & & 4,6 \\
\hline
\end{tabular}

SM: Salário Mínimo; *: Teste qui-quadrado; *: associação significativa; OR: Odds-Ratio; 27 adolescentes não responderam quanto ao consumo de bebida alcoolica; 52 não responderam quanto ao consumo de tabaco e 41 quanto ao uso de drogas ilícitas.

Em relação ao consumo de tabaco, associação significativa foi encontrada em relação à $8^{a}$ série $(p=0,0004)$, que apresentou uma probablidade de consumo de 4,6 vezes a probablidade na $7^{\mathrm{a}}$ série $(\mathrm{OR}=4,6)$ e predominio significativo naqueles com menor rendimento familiar ( $p<0,0001$ ), com razão de probablidade 71,3 vezes para um rendimento menor que um salário e 55 vezes para um a dois salários em relação à proporção de consumo entre os adolescentes com rendimento familiar acima de três salários mínimos. As prevalências foram similares para ambos os sexos, uma vez que apesar da prevalência ser maior para o sexo feminino, não se observou significância estatística.

Para o uso de drogas ilícitas, a significância estatística foi observada apenas em relação ao rendimento familiar, em que 14 (66,7\%) dos adolescentes com rendimento familiar igual ou superior a três salários mínimos, tinha 4,6 vezes de probablidade de utilização comparado com os adolescentes com rendimento inferior a um salário mínimo $(p=0,0176)$.

Tabela 3

Distribuição dos adolescentes de uma escola pública referente à frequencia de uso de bebida alcoolica, tabaco e drogras ilícitas em relação ao sexo, série, reprovação escolar e rendimento familiar. Uberaba, 2011.

\begin{tabular}{|c|c|c|c|c|c|c|c|}
\hline \multirow{2}{*}{$\begin{array}{l}\text { Frequência de uso } \\
\text { Álcool }\end{array}$} & & \multicolumn{2}{|c|}{ Diário ou Semanal } & \multicolumn{2}{|c|}{ Mensal ou Eventual } & \multirow[b]{2}{*}{$\mathrm{p}^{\#}$} & \multirow[b]{2}{*}{ OR } \\
\hline & & $\mathrm{n}$ & $\%$ & $\mathrm{~N}$ & $\%$ & & \\
\hline \multirow[t]{2}{*}{ Sexo } & Masc. & 28 & 57,1 & 21 & 42,9 & 0,2383 & 1,7 \\
\hline & Fem. & 14 & 43,8 & 18 & 56,3 & & 1 \\
\hline \multirow[t]{2}{*}{ Série } & $7^{\mathrm{a}}$ & 30 & 76,9 & 9 & 23,1 & $0,0001^{*}$ & 8,3 \\
\hline & $8^{\mathrm{a}}$ & 12 & 28,6 & 30 & 71,4 & & 1,0 \\
\hline \multirow[t]{2}{*}{ Reprovação } & $\operatorname{Sim}$ & 19 & 100 & 0 & 0 & $<0,0001 *$ & 65,6 \\
\hline & Não & 23 & 37,1 & 39 & 62,9 & & 1 \\
\hline \multirow[t]{3}{*}{ Rendimento Familiar } & $\leq 1 \mathrm{SM}$ & 22 & 91,7 & 2 & 8,3 & $<0,0001^{*}$ & 24,8 \\
\hline & 1 a 2 SM & 16 & 30,8 & 36 & 69,2 & & 1 \\
\hline & $\geq 3 \mathrm{SM}$ & 4 & 80 & 1 & 20 & & 9,0 \\
\hline \multicolumn{8}{|l|}{ Tabaco } \\
\hline \multirow[t]{2}{*}{ Sexo } & Masc. & 17 & 45,9 & 20 & 54,1 & 0,0689 & 4,3 \\
\hline & Fem. & 3 & 16,7 & 15 & 83,3 & & 1 \\
\hline \multirow[t]{2}{*}{ Série } & $7^{\mathrm{a}}$ & 14 & 53,8 & 12 & 46,2 & $0,0107 *$ & 4,8 \\
\hline & $8^{\mathrm{a}}$ & 6 & 20,7 & 23 & 79,3 & & 1,0 \\
\hline \multirow[t]{2}{*}{ Reprovação } & Sim & 4 & 57,1 & 3 & 42,9 & 0,4221 & 2,7 \\
\hline & RODRIGOE & ES DA SI & \multicolumn{5}{|c|}{ Revista de Enfermagem Referência - IV-n. ${ }^{\circ}$ 2-2014 } \\
\hline
\end{tabular}




\begin{tabular}{|c|c|c|c|c|c|c|c|}
\hline \multirow{4}{*}{ Rendimento Familiar } & Não & 16 & 33,3 & 32 & 66,7 & \multirow{4}{*}{0,3946} & 1 \\
\hline & $\leq 1 \mathrm{SM}$ & 10 & 47,6 & 11 & 52,4 & & 2,2 \\
\hline & 1 a 2 SM & 10 & 29,4 & 24 & 70,6 & & 1 \\
\hline & $\geq 3 \mathrm{SM}$ & 0 & 0 & 0 & 0 & & - \\
\hline \multicolumn{8}{|l|}{ Drogas Ilícitas } \\
\hline \multirow[t]{2}{*}{ Sexo } & Masc. & 3 & 13,6 & 19 & 86,4 & 0,5375 & 3,4 \\
\hline & Fem. & 0 & 0 & 9 & 100 & & 1 \\
\hline \multirow[t]{2}{*}{ Série } & $7^{\mathrm{a}}$ & 0 & 0 & 13 & 100 & 0,2452 & 1 \\
\hline & $8^{\mathrm{a}}$ & 3 & 16,7 & 15 & 83,3 & & 6,1 \\
\hline \multirow[t]{2}{*}{ Reprovação } & Sim & 0 & 0 & 9 & 100 & 0,5375 & 1 \\
\hline & Não & 3 & 13,6 & 19 & 86,4 & & 3,4 \\
\hline \multirow[t]{3}{*}{ Rendimento Familiar } & $\leq 1 \mathrm{SM}$ & 0 & 0 & 10 & 100 & $0,0267 *$ & 1 \\
\hline & 1 a 2 SM & 0 & 0 & 11 & 100 & & - \\
\hline & $\geq 3 \mathrm{SM}$ & 3 & 30 & 7 & 70 & & 9,8 \\
\hline
\end{tabular}

SM: Salário Mínimo; *:Teste qui-quadrado; ": associação significativa; OR: Odds-Ratio; 34 adolescentes não responderam quanto a frequência de consumo de bebidas alcoólicas; 21 não responderam quanto ao consumo de tabaco e 28 quanto a frequência de consumo de drogas ilícitas.

Na tabela 3, observa-se a distribuição das variáveis $\quad(p<0,0001 ; O R=24,8)$. Por sua vez, foi significativa estudadas quanto à frequência de consumo de álcool, a maior frequência de consumo de tabaco (diária ou cigarro e drogas. Houve predomínio da frequência de semanal) encontrada somente nos adolescentes da $7^{a}$ consumo do álcool mais elevada (diária ou semanal) série $(\mathrm{p}=0,0107 ; \mathrm{OR}=4,8)$. Houve maior frequência entre adolescentes da $7^{\mathrm{a}}$ série $(\mathrm{p}=0,0001 ; \mathrm{OR}=8,3)$, de uso de drogas ilícitas (diária ou semanal) naqueles sem reprovação escolar $(p<0,0001 ; O R=65,6)$ e com rendimento familiar superior a 3 salários mínimos com rendimento familiar de até um salário mínimo $\quad(p=0,0267 ; \mathrm{OR}=9,8)$.

Tabela 4

Resumo descritivo e comparativo da idade de incicio do uso de bebida alcoolica, tabaco e drogas ilícitas em relação ao sexo, série, reprovação e rendimento dos adolescentes de uma escola pública. Uberaba, 2011.

\begin{tabular}{|c|c|c|c|c|c|}
\hline Álcool & & $\mathrm{N}$ & Média & $\mathrm{EP}$ & $\mathrm{p}^{\#}$ \\
\hline \multirow[t]{2}{*}{ Sexo } & Masc. & 66 & 14,23 & 0,09 & 0,7408 \\
\hline & Fem. & 49 & 14,18 & 0,10 & \\
\hline \multirow[t]{2}{*}{ Série } & $7^{\mathrm{a}}$ & 39 & 13,64 & 0,09 & $<0,0001^{*}$ \\
\hline & $8^{\mathrm{a}}$ & 76 & 14,50 & 0,06 & \\
\hline \multirow[t]{2}{*}{ Reprovação } & $\operatorname{Sim}$ & 19 & 13,21 & 0,12 & $<0,0001$ * \\
\hline & Não & 96 & 14,41 & 0,05 & \\
\hline \multirow[t]{3}{*}{ Rendimento Familiar } & $\leq 1 \mathrm{SM}$ & 25 & 13,80 & 0,13 & $0,002 *$ \\
\hline & 1 a 2 SM & 69 & 14,23 & 0,08 & \\
\hline & $\geq 3 \mathrm{SM}$ & 21 & 14,62 & 0,14 & \\
\hline \multicolumn{6}{|l|}{ Tabaco } \\
\hline \multirow[t]{2}{*}{ Sexo } & Masc. & 41 & 13,83 & 0,11 & 0,5904 \\
\hline & Fem. & 35 & 13,91 & 0,11 & \\
\hline \multirow[t]{2}{*}{ Série } & $7^{\mathrm{a}}$ & 31 & 13,65 & 0,12 & $0,0165 *$ \\
\hline & $8^{\mathrm{a}}$ & 45 & 14,02 & 0,10 & \\
\hline \multirow[t]{2}{*}{ Reprovação } & $\operatorname{Sim}$ & 10 & 13,60 & 0,21 & 0,1822 \\
\hline & Não & 66 & 13,91 & 0,08 & \\
\hline \multirow[t]{3}{*}{ Rendimento Familiar } & $\leq 1 \mathrm{SM}$ & 27 & 13,70 & 0,13 & 0,1177 \\
\hline & 1 a 2 SM & 49 & 13,96 & 0,10 & \\
\hline & $\geq 3 \mathrm{SM}$ & 0 & - & - & \\
\hline \multicolumn{6}{|l|}{ Drogas Ilicitas } \\
\hline \multirow[t]{2}{*}{ Sexo } & Masc. & 38 & 13,92 & 0,12 & 0,9371 \\
\hline & Fem. & 21 & 13,90 & 0,16 & \\
\hline
\end{tabular}




\begin{tabular}{lccccc} 
Série & $7^{\mathrm{a}}$ & 22 & 13,59 & 0,15 & $0,0092 *$ \\
\multirow{2}{*}{ Reprovação } & $8^{\mathrm{a}}$ & 37 & 14,11 & 0,12 & 0,1203 \\
\multirow{3}{*}{ Rendimento Familiar } & Sim & 14 & 13,64 & 0,20 & 0,1293 \\
& Não & 45 & 14,00 & 0,11 & \\
& $\leq 1 \mathrm{SM}$ & 14 & 13,64 & 0,20 & 0,13 \\
\hline
\end{tabular}

": Diferença significativa; EP: Erro Padrão da Média; ": Teste ANOVA-f; SM: Salário Mínimo.

De acordo com a Tabela 4, a variável média de idade de início de utilização das substâncias estudadas, apresentou diferença significativa: dentre os 115 que responderam ter consumido álcool observou-se: idade média de início significativamente superior nos adolescentes da $8^{a}$ série $(p<0,0001)$, sem reprovação escolar $(p<0,0001)$ e com rendimento familiar igual ou maior que $3 \mathrm{SM}$ em relação aqueles com rendimento familiar inferior a $1 \mathrm{SM}(\mathrm{p}=0,0003)$.

\section{Discussáo}

De acordo com Silveira e Santos (2012), associa-se maior vulnerabilidade social à baixa condição socioeconómica como observada nestes dados, enquanto fator que pode contribuir para uma maior exposição a situações de risco, como abandono escolar, gravidez na adolescência, violência, uso de álcool, tabaco e drogas.

Destaca-se que estão associados ao uso de bebidas alcoólicas o aumento da violência intra e extrafamiliar, a ocorrência de acidentes de trânsito, o déficit e abandono escolar, comportamentos de risco como transmissão de DST, agressões, depressões clínicas e gravidez não planejada. Em estudo qualitativo realizado com adolescentes entre $12 \mathrm{e}$ 20 anos, identificou-se déficit no autocuidado dos adolescentes associado à maior exposição a situações de risco de vida inerente ao consumo abusivo de álcool, que ocorria, predominantemente, quando os jovens saíam em grupos de amigos (Silva, Padilha, \& Santos, 2011). Neste sentido, sugere-se a realização de ações educativas em saúde que despertem atitudes de autocuidado nos adolescentes a fim de mantê-los longe destes riscos.

Em estudo que considerou dados de adolescentes residentes nas 27 capitais brasileiras, foi observado que cerca de três quartos dos adolescentes de 13 a 15 anos já experimentaram álcool, cerca de um quarto bebeu regularmente nos últimos 30 dias com episódios de embriaguez e $9 \%$ relatam ter tido problemas com o álcool. Quanto às drogas, 8,7\% relataram já ter experimentado estas substâncias alguma vez na vida, sendo que a experiência com álcool e drogas ocorreu precocemente (Malta et al., 2011).

Os resultados encontrados para o consumo de tabaco vão de encontro à literatura que aponta um discreto predomínio entre as meninas (Horta et al., 2007; Malbergier et al., 2012). Estudo realizado com uma população de adolescentes entre 10 e 19 anos da região sul do Brasil, identificou que a prevalência de tabagismo na amostra foi de $12,1 \%$. Os fatores de risco para tabagismo, na análise multivariada, por regressão logística, foram: maior idade, odds ratio (OR) de 28,7, irmãos mais velhos fumem, OR de 2,4 três ou mais amigos que fumem, OR de 17,5 e baixa escolaridade OR de 3,5 (Malcon, Menezes, \& Chatkin, 2003).

Sobre o consumo de substâncias psicoativas, bem como uma série de outros comportamentos humanos, há que considerar algumas questões inerentes ao género, entre outras variáveis. Considera-se que os respondentes de sexos diferentes estiveram expostos a diferentes situações familiares, sociais e outras, que configuram distintos padrões de contacto com cada um dos grupos de substâncias e isto pode estar relacionado com condições históricas e sociais, determinadas pelo modo como se constroem os conceitos de masculino e feminino. Além disso, cada vez mais o consumo de tais substâncias se equiparam entre meninos e meninas, o que representa um agravamento na saúde pública (Malbergier et al., 2012).

Para melhor aferir os indicadores do comportamento dos sujeitos em relação ao álcool, as variáveis uso na vida e uso regular (beber pelo menos três dias na semana) têm sido incorporadas nas pesquisas da área. Neste estudo, trabalhou-se com a ocorrência de consumo no mês que antecedeu as entrevistas, cujos dados corroboram com o estudo de Horta et al. (2007), que encontrou prevalência de 49\% para o sexo masculino e $37,9 \%$ para o feminino. Outro estudo, 
que incluiu 499 adolescentes de Cuiabá (Brasil), encontrou dados distintos, em que as meninas $(52,4 \%)$ consomem mais bebidas alcóolicas que os meninos (45,2\%); contudo o início do consumo da bebidas alcoólicas e do tipo mais consumido estão de acordo com estes dados (Jesus et al., 2011).

Estudo de revisão que avaliou a eficácia dos programas de intervenção para a prevenção de substâncias psicoativas, dirigidos a adolescentes dos 10 aos 16 anos de idade em meio escolar, observou que, embora a base conceptual dos programas seja relativamente uniforme, verifica-se uma grande variedade nas intervenções e na sua implementação, contudo, a maioria dos programas são eficazes no aumento dos conhecimentos acerca do consumo de álcool e alguns desenvolvem atitudes e expectativas mais seguras acerca de sua utilização entre jovens. (Barroso, Barbosa, \& Mendes, 2006).

Podem ser elencados vários fatores que condicionam o consumo de álcool nesta idade, dos quais se destacam: frágil contexto familiar e social - a utilização por pais ou familiares e baixo custo e fácil acesso - influência dos media que associa o álcool a situações de prazer e bem-estar e por uma larga inserção em estabelecimentos comerciais, festas, casas noturnas, postos de gasolina e quiosques perto da escola, fatores que podem contribuir para um estágio de dependência na idade adulta (Vieira, Ribeiro, Romano, \& Laranjeira, 2007).

Para o tabagismo na adolescência, a considerar os 76 participantes deste estudo, os resultados permitem inferir que os adolescentes que frequentam a $7^{\mathrm{a}}$ série iniciam o consumo nesta época, a exemplo dos que estão no oitavo ano, que experimentam o cigarro ao ingressar nesta série. Tais dados corroboram com os resultados da literatura, que apontam a idade de início de consumo de tabaco entre 13 e 15 anos e maior prevalência de uso na faixa dos 17 a 19 anos para ambos os sexos (Malco et al.,2003).

A mesma relação que aponta a idade média significativamente superior nos adolescentes da $8^{\text {a }}$ série observa-se para as drogas ilícitas. No estudo de Jesus et al. (2011), considerando a utilização de drogas ilícitas entre adolescentes, identificou que 10,5\% dos meninos (Idade de início aos 15 anos) e 5,8\% das meninas (início aos 16 anos) consumiram drogas, dos quais, 28,9\% já fizeram uso de cocaína, bem como mais de uma droga, e 15,7\% utilizaram maconha. A maioria dos meninos (60,0\%) e 33,3\% das meninas consumiam droga ocasionalmente $(\mathrm{p}=$ 0,0162).

Entre as drogas utilizadas na análise deste estudo, observa-se distribuição semelhante entre os sexos, em que o sexo feminino tem menor média de idade para o início de consumo de álcool e drogas ilícitas, facto que pode representar um contacto mais precoce do sexo feminino com estas substâncias ou pode começar a ser percebido um movimento que levará ao estabelecimento de padrões de consumo de drogas, em maiores proporções para o sexo feminino em gerações futuras. Qualquer dos dois cenários, que parecem não ser eliminatórios entre si, implica uma preocupação imediata do ponto de vista da saúde pública, mais especificamente, do ponto de vista da saúde da mulher.

Ressalta-se a necessidade de suporte familiar e acompanhamento escolar adequado destes jovens para eliminar as situações de vulnerabilidade a que estes jovens estão expostos, em especial na comunidade que fora objeto desta investigação (Cavalcante, Alves, \& Barroso, 2008).

Um outro fator importante refere-se ao consumo de drogas entre os familiares, que associado a outros fatores de risco (psicológicos e socioculturais) dos adolescentes, como: situação socioeconómica da família, dificuldades no envolvimento familiar (problemas de relacionamento, conflitos, ausência e falta de apoio dos pais), violência doméstica, amigos que consomem droga, evasão, insucesso e abandono escolar, falta de opções de lazer e recreio, ausência de apoio social e religioso, entre outros, são fatores condicionantes ao maior consumo de drogas pelos estudantes adolescentes, em diversos contextos socioculturais, como destacado por vários autores (Inglés et al., 2007; Suzuki et al., 2008; Jinez et al., 2009; Naude et al., 2011; Coomber et al., 2012).

Algumas iniciativas têm dado enfoque aos temas (uso de álcool, tabaco e drogas, além de outras situações de risco) ao inserir estratégias de educação em saúde na escola, voltadas para os adolescentes, como o projeto de extensão ligado à esta pesquisa (Silveira et al., 2011).

A educação permanente de professores para o lidar com estes adolescentes também está a ser consolidada como estratégia de prevenção destas condutas de risco, sendo desenvolvidas especialmente pelo Enfermeiro profissional, que possui como uma de suas principais atribuições a Educação em Saúde, 
direcionada para o autocuidado (Silveira, Reis, Santos, Borges, \& Fonseca, 2012).

\section{Conclusão}

Em resposta aos objetivos desta investigação, afirmase que o uso de bebidas alcoólicas e tabaco está direta e significativamente associado à 8 a série, à não reprovação escolar ao maior rendimento familiar. Quanto ao consumo de drogas a associação foi significativa apenas para o grupo de maior poder económico. Resultados semelhantes foram obtidos nas associações das variáveis em comparação à frequência e à idade de início do consumo de drogas, lícitas ou ilícitas. Não foi observada diferença significativa do ponto de vista estatístico para as variáveis de género, o que sugere um padrão de consumo razoavelmente semelhante entre meninos e meninas para as classes de drogas estudadas. Destaca-se a alta proporção de estudantes que não responderam as questões específicas sobre consumo de drogas, facto que pode significar que os resultados descritos estejam subestimados.

Os resultados deste estudo refletem a necessidade de estratégias de educação em saúde, direcionadas para adolescentes, observando a inserção de metodologias mais inclusivas, direcionadas e lúdicas, estimulando a reflexão crítica destes alunos acerca das situações de risco e vulnerabilidades relacionadas ao seu comportamento sexual, consumo de bebidas alcóolicas, tabaco e drogas ilícitas. Cabe considerar que estes resultados referentes a vulnerabilidades fazem parte de uma pesquisa que antecedeu a abordagem destas temáticas em oficinas de prevenção, no desenvolvimento do projeto de Extensão citado anteriormente.

Há que se priorizar ainda a capacidade do docente que está em contacto com estes alunos, fornecendo ao profissional de educação, ferramentas para a abordagem destas questões, entre outras, apoiado pela equipa das unidades de atenção primária à saúde de referência local.

Este estudo tem como limitações o facto de ter sido desenvolvido numa única escola de uma cidade e estado brasileiro. Contudo os seus resultados podem possibilitar inferências uma vez que a realidade local se aproxima ao cenário encontrado em diversas regiões e capitais do país.
Ressalta-se por fim, ao considerar as carências sociais, económicas e culturais da população estudada, a necessidade de maior articulação entre os setores de Segurança, Seguridade Social, Educação e de Saúde, para trabalharem em conjunto na redução dos riscos de vulnerabilidade inerentes à complexidade da temática adolescente. Neste sentido, fica apresentado o papel da Universidade, que, ao trabalhar a pesquisa, permite uma compreensão da realidade local o que pode levar à proposta de estratégias de intervenção sob a forma de programa de extensão universitária.

\section{Referências bibliográficas}

Agresti, A. (2007). An introduction to categorical data analysis (2nd ed.). Hoboken, NJ: John Wiley.

Barroso, T., Barbosa, A., \& Mendes, A. (2006). Programas de prevenção do consumo de álcool em jovens estudantes Revisão sistemática. Revista de Enfermagem Referência, 2 (3), 33-44.

Cavalcante, M. B. P. T., Alves, M. D. S., \& Barroso, M. G. T. (2008). Adolescência, álcool e drogas: Uma revisão na perspectiva da promoção da saúde. Escola Anna Nery Revista de Enfermagem, 12 (3), 555-559.

Coomber, K., Toumbourou, J. W., Miller, P., Staiger, P. K., Hemphill, S. A., \& Catalano R. F. (2012). Rural adolescent alcohol, tobacco, and illicit drug use: A comparison of students in Victoria, Australia, and Washington State, United States. The Journal of Rural Health, 27 (4), 409-415.

Govindarajuju, Z. (2007). Nonparametric inference. Hackensack, NJ: World Scientific Publishing.

Horta, R. L., Horta, B. L., Pinheiro, R. T., Morales, B., \& Strey, M. N. (2007). Tobacco, alcohol, and drug use by teenagers in Pelotas, Rio Grande do Sul State, Brazil: A gender approach. Cadernos de Saúde Pública, 23 (4), 775-783.

Inglés, C. J., Delgado, B., Bautista, R., Torregrosa, M. S., Espada, J. P., Carcía-Fernández, J. M., García-López, L. J. (2007). Factores psicosociales relacionados con el consumo de alcohol y tabaco en adolescentes españoles. International Journal of Clinical and Health Psychology, 7 (2), 403-420.

Jesus, F. B., Lima, F. C. A., Martins, C. B. G., Matos, K. F., \& Souza, S. P. S. (2011). Vulnerabilidade na adolescência: A experiência e expressão do adolescente. Revista Gaúcha de Enfermagem, 32 (2), 359-367.

Jinez, M. L. J., Souza, J. R. M., \& Pillon, S. M. (2009). Uso de drogas e fatores de risco entre estudantes de ensino médio. Revista Latino-Americana de Enfermagem, 17(2), 246-252.

Malbergier, A., Cardoso, L. R. D., Amaral, R. A., \& Santos, V. C. V. (2012). Gender parity and drug use: Are girls catching up with boys? Revista Brasileira de Psiquiatria, 34 (1), 16-23. 
Malcon, M. C., Menezes, A. M. B., \& Chatkin, M. (2003). Prevalência e fatores de risco para tabagismo em adolescentes. Revista de Saúde Pública, 37 (1), 1-7.

Malta, D. C., Porto, D. L., Melo, F. C. M., Monteiro, R. A., Sardinha, L. M. V., \& Lessa, B. H. (2011). Family and the protection from use of tobacco, alcohol, and drugs in adolescents, National School Health Survey. Revista Brasileira de Epidemiologia, 14 (Supl. 1), 166-177.

Naude, C. E., Senekal, M., Laubscher, R., Carey, P. D., \& Fein, G. (2011). Growth and weight status in treatment-naive 12-16 year old adolescents with alcohol use disorders in Cape Town, South Africa. Nutrition Journal, 10 (1), 87.

Pedrosa, A. A. S., Camacho, L. A. B., Passos, S. R. L., \& Olveira, R. V. C. (2011). Consumo de álcool entre estudantes universitários. Cadernos de Saúde Pública, 27(8), 1611-1621.

Silva, S. E. D., Padilha, M. I. C. S., \& Santos, L. M. S. (2011). A enfermagem estimulando o autocuidado de adolescentes a partir das representações sociais desses sobre as bebidas alcoólicas. Enfermagem em Foco, 2 (3), 160-163.
Silveira, R. E., Reis, N. A., Santos, A. S., \& Borges, M. R. (2011). Qualidade de vida de docentes do ensino fundamental de um município Brasileiro. Revista de Enfermagem Referência, 3 (4), 115-123.

Silveira, R. E., Reis, N. A., Santos, A. S., Borges, M. R., \& Fonseca, A. S. (2012). Workshops with teachers: Health education for management with adolescents. Acta Paulista de Enfermagem, 25 (Spe. 2), 169-174.

Silveira, R. E., \& Santos, A. S. (2012). Contextos de vulnerabilidade entre adolescentes do ensino fundamental de Uberaba/MG. Enfermagem em Foco, 3 (4), 182-185.

Suzuki, K., Kimura, M., Takeda, A., \& Matsushita, S. (2008). Is adolescent tobacco use a gateway drug to adult alcohol abuse? A Japanese longitudinal prospective study on adolescent drinking. Nibon Arukoru Yakubutsu Igakkai Zasshi, 43 (1), 44-53.

Vieira, D. L., Ribeiro, M., Romano, M., \& Laranjeira, R. R. (2007). Álcool e adolescentes: Estudo para implementar políticas municipais. Revista de Saúde Pública, 41 (3), 396-403. 